\title{
La Généalogie des dieux païens entre le Décaméron et les nouvelles des humanistes du premier $\mathrm{XV}^{\mathrm{e}}$ siècle
}

\section{Pauline Pionchon}

\section{OpenEdition}

\section{Journals}

Édition électronique

URL : http://journals.openedition.org/cei/167

DOI : $10.4000 /$ cei. 167

ISSN : 2260-779X

\section{Éditeur}

UGA Éditions/Université Grenoble Alpes

\section{Édition imprimée}

Date de publication : 15 mars 2010

Pagination : $55-78$

ISBN : 978-2-84310-164-9

ISSN : $1770-9571$

\section{Référence électronique}

Pauline Pionchon, «La Généalogie des dieux païens entre le Décaméron et les nouvelles des humanistes du premier xve siècle ", Cahiers d'études italiennes [En ligne], 10 | 2010, mis en ligne le 15 septembre 2011, consulté le 27 mars 2021. URL : http://journals.openedition.org/cei/167 ; DOI : https://doi.org/ $10.4000 /$ cei. 167 


\title{
LA GÉNÉALOGIE DES DIEUX PAÏENS ENTRE LE DÉCAMÉRON ET LES NOUVELLES DES HUMANISTES DU PREMIER XV ${ }^{\mathrm{e}}$ SIÈCLE
}

\author{
Pauline Pionchon
}

Université de Strasbourg

La Genealogia Deorum gentilium est un imposant répertoire mythologique traitant des personnages qui peuplent la poésie de l'ancienne Grèce et de la Rome antique. Boccace clôt cet ouvrage d'érudition par deux livres (XIV et $\mathrm{XV}$ ) dans lesquels il entend défendre contre ses détracteurs la poésie antique. Cette défense des poètes païens s'inscrit dans une tradition polémique qui commence en Italie avec les textes d'Albertino Mussato, puis de Pétrarque, et que de nombreux autres humanistes prolongeront aux $\mathrm{Xv}^{\mathrm{e}}$ et $\mathrm{XVI}^{\mathrm{e}}$ siècles ${ }^{\mathrm{I}}$. Reprenant certaines des idées soutenues par Mussato et par Pétrarque dans des écrits ponctuels ${ }^{2}$, y adjoignant des apports personnels ${ }^{3}$, le plaidoyer de Boccace repose sur une redéfinition de la poésie, dont la portée ne se limite pas aux écrits de l'Antiquité.

Les témoignages de Filippo Villani et Leonardo Bruni nous apprennent que les humanistes du premier $\mathrm{Xv}^{\mathrm{e}}$ siècle, qui considéraient Boccace

I. A. Stäuble, "Francesco da Fiano in difesa della poesia", Bibliothèque d'Humanisme et Renaissance, I964, XXVI, p. 256-259; G. Ronconi, "Giovanni Dominici e le dispute sulla poesia nel primo umanesimo", dans Dizionario Critico della letteratura italiana, Turin, UTET, 1973; Idem, Le origini delle dispute umanistiche sulla poesia (Mussato e Petrarca), Rome, Bulzoni, 1976; C. Mésoniat, Poetica theologia. La "Lucula noctis" di Giovanni Dominici e le dispute letterarie tra '300 e '400, Rome, Edizioni di Storia e Letteratura, 1984.

2. Albertino Mussato contribua à cette dispute par ses lettres à Giovanni da Vigonza (In laudem poeticae, ad d. Ioannem de Viguntia simulantem se abhoruisse seria Priapeiae), au Collegio degli Artisti de Padoue à l'occasion de son couronnement (Ad Collegium Artistarum), à un grammairien vénitien (Ad Ioannem, grammaticae professorem, docentem Venetiis), au dominicain Giovannino da Mantova. Les écrits par lesquels Pétrarque participa à la défense de la poésie antique sont : Familiares, X, 4 (lettre à son frère Gherardo); Seniles, XV, in (lettre à Benvenuto da Imola); Invectivae contra medicum, III; De sui ipsius et aliorum ignorantia; à ces textes on peut ajouter la Collatio laureationis.

3. Par ailleurs, Giuseppe Billanovich a consacré un article aux apports de Pietro Piccolo da Monteforte pour les derniers livres de la Généalogie (G. Billanovich, "Pietro Piccolo da Monteforte tra il Petrarca e il Boccaccio ", dans Medioevo e Rinascimento. Studi in onore di Bruno Nardi, I, I955, p. 3-76). 
comme un guide tutélaire de leurs études, ont non seulement lu, mais aussi admiré la Généalogie des Dieux païens, une œuvre qui, de l'avis de tous, écrit Giannozzo Manetti, est le chef-d'œuvre de Boccace. On sait aussi que les livres XIV et XV ont rapidement circulé de façon autonome dans les milieux humanistes, en deçà et au-delà des Alpes ${ }^{4}$, et que les défenses de la poésie de nombreux auteurs, dont Coluccio Salutati, en sont inspirées's. Dans le cadre d'une étude sur le genre de la nouvelle au $\mathrm{Xv}^{\mathrm{e}}$ siècle, il sera donc intéressant de voir en quoi la conception de la poésie exprimée dans la Généalogie a influencé la pratique de ce genre chez les humanistes. Dans quelle mesure la Généalogie a-t-elle eu une fonction de filtre entre le principal modèle du genre, le Décaméron, et les nouvelles de Leonardo Bruni, Leon Battista Alberti, Enea Silvio Piccolomini?

En premier lieu, nous reviendrons sur la conception de la poésie dans les derniers livres de la Généalogie, puis sur une question qui divise la critique, à savoir le problème de la réception du Décaméron par son propre auteur, deux décennies après la rédaction de l'ouvrage.

\section{Le Décaméron à l'épreuve de la Généalogie des dieux païens}

Nous ne reprendrons pas tous les thèmes traités dans les derniers livres de la Généalogie, où bien des assertions contradictoires se côtoient; mais nous limiterons notre commentaire aux aspects les plus pertinents pour notre propos. Rappelons-nous qu'il s'agit d'un ouvrage longtemps retravaillé et demeuré inachevé. On peut ramener les contradictions qui le parcourent à plusieurs facteurs, dont la forme choisie par Boccace et la phénoménologie de la construction de la pensée à travers la réévaluation et le dépassement de la conception médiévale de la poésie ${ }^{6}$. Boccace a conçu ces livres comme une réponse aux détracteurs de la poésie des auteurs païens :

4. G. Di Stefano, "Il Trecento», dans C. Pellegrini (éd.), Il Boccaccio nella cultura francese, Florence, Olschki, I97I, p. I-47; L. Sozzi, «Boccaccio in Francia nel Cinquecento », ibidem, p. 2II-349.

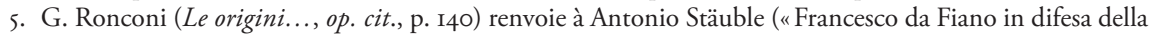
poesia", art. cité), lequel mentionne parmi les auteurs ayant participé à cette dispute par leurs écrits, Coluccio Salutati (De laboribus Herculis, I; Epistolario, IV, I5; I8; X, I6; XII, 20 [lettre à Giovanni da San Miniato]; 24 [lettre à Giovannino Dominici]), Francesco da Fiano (Contra ridiculos obluctores et fellitos detractores poetarum), Pier Paolo Vergerio (Epistolario, LXXXI), Leonardo Bruni (traduction de l'Oratio ad adolescentes de Basile de Césarée; De studiis et litteris), Giovanni di Balbo da Faenza (Tractatus qui concluditur nulla gentilium scientiam fidei christiane esse contrariam), Ciriaco d'Ancona (lettre de I423), Maffeo Vegio (De educatione liberorum et eorum claris moribus), Enea Silvio Piccolomini (De liberorum educatione; lettre à Ladislas de Hongrie, de I450), Guarino da Verona (lettre à Giovanni da Prato), Timoteo Maffei (In sanctam rusticitatem litteras impugnantem), Raffaele da Parnassio (De consonantia naturae et gratiae).

6. F. Tateo, "Retorica" $e$ "poetica" fra Medioevo e Rinascimento, Bari, Adriatica, 1960, p. 67-I60. 
il présente donc méthodiquement chaque type d'adversaire, puis expose ses arguments contre la poésie, avant d'y répondre en présentant, tout aussi méthodiquement, ses propres raisons. D'une part, une telle organisation du discours occasionne des répétitions qui permettent à l'auteur de nuancer ses propos, puisant dans des traditions différentes, à quelques pages d'intervalle; d'autre part, elle impose à la pensée de se construire en réaction face aux attaques et aux arguments des adversaires. La nécessité de répliquer et de convaincre amènent l'auteur à déployer les nombreuses facettes "disjointes» d'une pensée non encore unifiée - d'une pensée en train de se construire.

L'argumentation de Boccace en faveur de la poésie des Anciens repose essentiellement sur son identification à la fabula - et c'est là son aspect le plus moderne ${ }^{7}$.

Les détracteurs de la poésie des Anciens soutenaient notamment que la poésie antique racontait des histoires invraisemblables, qui n'étaient porteuses d'aucune signification profonde. Boccace défend au contraire l'idée que la poésie est une discipline cognitive et que sous l'écorce («cortex») de la fiction circule le suc du récit : dans un mouvement paradoxal, le poète inspiré révèle des vérités profondes en les voilant de fiction, fondant son écriture dans les procédés de l'allégorie (XIV, 7 et I2). Les Bucoliques de Virgile, mais aussi celles de Pétrarque en sont d'illustres exemples; le Buccolicum carmen de Boccace en est un autre, dont il a lui-même fait l'exégèse dans sa lettre à Martino da Signa, comme il prend soin de le rappeler (XIV, Io). Le contenu de ces fables allégoriques sont de teneur philosophique, voire métaphysique, puisqu'elles émanent d'une méditation sur le cosmos.

Cette conception de la fabula comme fable allégorique voilant et dévoilant l'ordre de la nature, qui représente probablement aux yeux de Boccace la forme la plus noble de poésie, est souvent mise en avant. Sa prédominance dans l'argumentation ne doit pourtant pas faire oublier qu'en réalité, le terme "poésie» recouvre pour Boccace une littérature beaucoup plus vaste. L'ensemble des livres XIV et XV fait clairement apparaître que la notion de poésie qu'il défend ne se limite pas à la fable allégorique modelée sur la poésie bucolique ou la fable ésopique. Les limites du genre dont il traite sont mouvantes : elles varient d'un chapitre à l'autre, tantôt dans le sens de la restriction, tantôt dans celui de l'élargissement.

7. Ibid., p. I27. Tateo précise que cette identification n'est pas en elle-même absolument nouvelle : la nouveauté réside dans le sens que Boccace donne au terme fabula, à travers l'inclusion et l'exclusion d'aspects traditionnels de sa définition. 
Les chapitres 9 et io du livre XIV témoignent d'une conception des moins restrictives de la poésie. En se basant sur le double critère de la vérité et de l'imagination, ainsi que sur celui des modes possibles d'interprétation, Boccace distingue quatre types de fabulae. La première catégorie correspond aux fables allégoriques, dont la lettre n'est pas vraisemblable ${ }^{8}$. La seconde regroupe les fictions qui mêlent vérité et fabuleux, suivant le modèle des Métamorphoses d'Ovide : le poète recourt ici à l'allégorie et à l'imitation de la nature9. La troisième catégorie rassemble les poèmes épiques et les pièces des comiques "moraux», Plaute et Térence. Contrairement aux poèmes épiques, qui mélangent une matière historique et des éléments fictifs, et recourent à l'imitation de la nature et à l'allégorie ${ }^{10}$, les écrits des comiques relèvent uniquement de l'imitation :

[ils] ne donnent rien à comprendre que ce que dit la lettre, mais ils veulent par leur art décrire les mours et les propos des humains, éduquer ainsi les lecteurs et les mettre sur leurs gardes. Si ces faits ne sont pas réels, ils ont pu l'être, car ils sont ordinaires. (XIV, 9)

Ce type de fabula - récit de faits non advenus mais vraisemblables - a une valeur métonymique : il ne s'agit pas pour le lecteur de découvrir un sens voilé, mais bien plutôt, en allant du plan de l'anecdote individuelle à celui de la vérité générale, de mesurer la portée de la leçon de telles fictions - lesquelles se trouvent justifiées par leur caractère exemplaire, c'est-àdire par leur dimension cognitive et didactique ${ }^{\text {II }}$. Comme le fait remarquer Francesco Tateo, «l'intentio du poète pouvait donc aussi s'arrêter à la

8. XIV, 9: «Dans la première [sorte], l'écorce manque totalement de vérité, comme par exemple quand on fait parler entre eux des animaux ou des objets. L'auteur le plus grand en est Ésope [...]. » Nos extraits de la Généalogie sont tirés de G. Boccaccio, La Généalogie des Dieux païens, livres XIV et XV. Un manifeste pour la poésie, traduit, présenté et annoté par Y. Delègue, Strasbourg, Presses Universitaires de Strasbourg, $200 \mathrm{.}$. On peut lire le texte original latin dans G. Boccaccio, Tutte le opere (V. Branca éd.), Milan, Mondadori, I998, vol. VII-VIII.

9. XIV, 9 : «Dans la seconde sorte, la surface mêle des éléments fabuleux à la vérité, comme lorsque nous racontons que les filles de Minée ont été changées en chauve-souris, pour avoir méprisé les fêtes de Bacchus tandis qu'elles tissaient, ou que les compagnons d'Acetès le marin, qui projetaient d'enlever Bacchus, furent changés en poissons. Ces fables, les poètes les plus anciens du premier âge les ont inventées : leur intention était d'habiller de fictions aussi bien les choses humaines que divines."

IO. XIV, 9: «La troisième sorte est plus proche de l'histoire que de la fable. Les poètes célèbres en ont usé différemment. Même s'ils semblent écrire l'histoire, les poètes épiques, Virgile par exemple, quand il raconte la tempête qui secouait Énée sur la mer, ou Homère quand il décrit Ulysse attaché au mât de son navire pour ne pas être entraîné par le chant des sirènes, signifient sous ce voile tout autre chose que ce qu'ils montrent. "

II. XIV, 9 : "Condamner la troisième sorte (mais c'est impossible), reviendrait à condamner celle dont a usé le plus souvent le Christ Jésus, fils de Dieu, notre sauveur, quand il était dans notre chair. Certes les saintes Lettres n'emploient pas le terme dont se servent les poètes : certains disent "parabole», d'autres "exemple", parce que cette parole < vaut > par la raison de l'exemple." (C'est nous qui soulignons.) 
lictera, mais cette lictera devait porter en elle le signe d'une réflexion sur le monde des hommes ${ }^{12} »$.

À ces trois catégories de récit, Boccace en adjoint une quatrième, qu'il mentionne pour la disqualifier : ce sont les récits ineptes que les vieilles femmes racontent aux veillées; ils sont conçus sans savoir-faire et ne sont porteurs d'aucune vérité, ni dans la lettre ni au-delà ou en dessous de celle-ci ${ }^{13}$. Dans le chapitre io du même livre, cependant, Boccace va jusqu'à nier l'existence de récits creux, privés de suc :

[...] il n'est pas une petite vieille, si folle soit-elle, qui, lorsqu'autour du foyer domestique dans les veillées d'hiver elle imagine et raconte les fables d'Orcus, des Fées, des Lammies, etc. (c'est ce dont le plus souvent sont faites leurs inventions), il n'en est pas une qui ne sache que ce qu'elle raconte est prétexte à quelque signification proportionnée aux forces modestes de son intelligence, et il n'y a rien là qui prête à rire. Grâce à quoi elle cherche à faire peur aux tout petits, à donner du plaisir aux fillettes, à distraire les vieillards, à montrer en tout cas la puissance de Fortune. (XIV, Io. C'est nous qui soulignons.)

Il apparaît que la conception de la fabula que Boccace développe - non pas ornement rhétorique, mais langage humain - embrasse un vaste champ. Puisque toute fiction a une dimension gnoséologique, la notion de poésie englobe tout récit faisant intervenir la fiction - dont la présence distingue la poésie de la rhétorique, comme Boccace l'écrit au chapitre 7 du livre $\mathrm{XIV}^{\mathrm{I}}$. Et si la fiction est le trait définitoire de la poésie, c'est en vertu du fait que toute fiction est le voile («velamen») d'une vérité générale, que l'allégorie comme invention est une espèce travestie de la mimesis.

Par ailleurs, si comme on l'a vu, Boccace semble parfois inscrire au nombre des fabulae signifiantes et dignes l'ensemble des récits de fiction, il trace néanmoins, de façon approximative, une sorte de ligne de démarcation entre les ouvrages moraux, qui relèvent réellement de la poésie, et les ouvrages immoraux, afin que les premiers soient exempts de la condamnation qui frappe justement les seconds (XIV, 6). Il indique comme domaine de prédilection de la poésie celui de la "théologie naturelle», à savoir «[des] phénomènes naturels, [des] mœurs et les actions des hommes illustres, parfois aussi [de] ce qui concerne les dieux; notamment dans les

I2. F. Tateo, "Retorica» e "poetica»..., op. cit., p. I33.

I3. XIV, 9: "Quant à la quatrième sorte, elle ne contient de vérité ni dans sa surface ni dans sa profondeur; c'est une invention de vieilles en délire. [...] Ils peuvent bien condamner la quatrième sorte parce que nul principe acceptable ne semble la commander, nul apport de l'art la fortifier, nul dessein ordonné la guider. Peu me chaut! Elle n'a rien à voir avec les fables des poètes, même si je crois que nos contradicteurs pensent qu'il n'y a entre elles nulle différence."

I4. "La rhétorique a elle aussi ses inventions, mais cacher le vrai sous le couvert des fictions n’est pas du rôle de la rhétorique. Est pure poésie toute composition usant d'un voile, tout ouvrage écrit avec art.» 
hymnes qu'ils ont composés au début pour louer leurs dieux et où ils ont caché leurs exploits sous l'écorce poétique $[\ldots]$ » (XV, 8). Il exalte également la satire, qui, pas moins que l'épopée de Virgile, mais avec une plus grande vigueur, incite à la vertu (XIV, I5). Bien qu'il écrive que « [...] sous le libertinage, sous les sottises [...] sont enfermées de savoureuses significations morales [...]», il concède aux détracteurs de la poésie que des poèmes "chantent les charmes des dieux païens et incitent à commettre leurs erreurs" (XIV, 6), et que les actions immorales de ces dieux sont haïssables, et les poètes qui les racontent tout autant (XIV, I4). Les poètes visés, qui sont «la lie de la poésie», sont les auteurs comiques (XIV, I4, I5, 19), dont quelques-uns se firent les auteurs immoraux de "fictions dégradantes» (XIV, I9) ${ }^{15}$, poussés par l'appât du gain (XIV, 6, I9) ou aiguillés soit par le désir d'être reconnus par un peuple débauché (XIV, I5, 19), soit simplement par leur disposition d'esprit (XIV, I9). Sont également exclues de l'horizon de la poésie véritable L'Art d'aimer et d'autres œuvres mineures d'Ovide, dont le talent est remarquable, mais «également voluptueux", et dont les conseils sont funestes (XIV, I5) ${ }^{\mathrm{I}}$, car elles chassent la vertu et dérèglent les mœurs (XIV, I9). Ce sont ces auteurs que Platon entend exclure de la cité (XIV, 9). Les œuvres des poètes qui "n'ont pas respecté la morale nécessaire au savoir-faire poétique» doivent donc être écartées (XIV, I5) ${ }^{17}$. L'obscénité ${ }^{18}$ et l'indécence sont, au moins officiellement, évacuées de l'horizon de la poésie qu'il convient de lire et d'écrire. Le jugement que Boccace porte sur les satires, de Juvénal en particulier, révèle que l'indécence, cependant, tient moins à l'objet traité qu'au regard que l'auteur porte sur cet objet, qu'à l'intention dans laquelle il traite un sujet licencieux. Considérons l'exemple de la sixième satire de Juvénal,

15. Cet argument est d'une grande commodité, car il s'affiche comme une concession aux adversaires des auteurs païens, mais on ne voit pas bien qui sont ces comiques immoraux, puisque l'on ne possède pas de texte de ces auteurs.

I6. Boccace précise que les mauvais conseils d'Ovide aux amants sont désuets, car les jeunes gens contemporains connaissent bien d'autres ruses : « [...] de nos jours, il n'y a pas de garçon assez fou ou de demoiselle assez simplette, si le désir lubrique meut leur cervelle, qui pour parvenir à leurs fins, ne savent des manœuvres plus fines que celles enseignées par celui qu’on croit être un maître éminent en la matière.» Yves Delègue remarque qu'il y a peut-être là un clin d'œil au Décaméron, où les jeunes pouvaient "en apprendre un peu plus sur l'art d'aimer." (Boccace, Généalogie, op. cit., n. 134, p. 94.)

I7. Précisément, Boccace écrit que ces poètes doivent "parfois" être écartés. L’adverbe temporel est peut-être une marque de l'absence d'une conviction absolue de Boccace en la matière, d'un avis en tout cas plus nuancé que le rejet qu'il prône par ailleurs.

I8. Dans le chapitre 6 du livre XIV, Boccace fait un parallèle entre les poètes licencieux et les sculpteurs obscènes : «Mais, de grâce, parce que Praxitèle ou Phidias, ces grands maîtres de la sculpture, ont sculpté Priape l'impudique en train de bander pour Iole, plutôt que Diane qu'on peut regarder en toute honnêteté [...], dirons-nous que ces arts sont à condamner?» L'argument renvoie à Priape, sujet précisément du poème qui avait valu à Mussato la désapprobation de Giovanni da Vigonza, ce qui avait été l'occasion de sa première épître en défense de la poésie (voir note 2, et G. Ronconi, Le origini, op. cit., p. 19-27). 
que Boccace connaissait bien pour s'en être amplement inspiré dans son Corbaccio. Pour dénoncer l'immoralité des femmes, l'auteur latin invente de très brèves saynètes de débauche féminine : la matière est obscène, mais la satire ne l'est pas, car l'intention du moraliste est explicite.

Dans la Généalogie, la dimension cognitive et édifiante de la fabula est primordiale, car elle en justifie l'écriture et en autorise la lecture. La beauté de l'écorce doit séduire le lecteur, c'est-à-dire l'inciter, par le plaisir de la lecture, à s'approcher des vérités que la fable renferme ${ }^{19}$. Pour autant, l'attrait de la fable ne demeure pas à proprement parler dans une position ancillaire par rapport à la dimension gnoséologique. Contrairement à l'orateur, qui puise des images dans un répertoire conventionnel, le poète imagine des inventions nouvelles ${ }^{20}$ : la parole poétique révèle le réel, elle doit avoir une incidence sur la perception du monde et la façon d'être au monde du lecteur. Et «[la] saveur [des vers] tient à l'étonnante sève de leurs pensées» (XIV, I9).

La Généalogie traite en premier lieu de la fabula des Anciens, mais la nouvelle conception de la poésie qui s’y déploie déborde le cadre de l'Antiquité. Cette évidence pousse la critique à se demander si les œuvres narratives de jeunesse de Boccace, et particulièrement le Décaméron, sont à mettre au nombre des fabulae, ou si la conception de la poésie de la Généalogie implique leur rejet hors du domaine poétique.

Dans son ouvrage Boccaccio. L'invenzione della letteratura mezzana, Francesco Bruni a démontré qu'entre le moment où Boccace écrit le Décaméron et les années qu'il consacre à sa défense de la poésie, sa conception de la culture a radicalement changé ${ }^{21}$. Selon lui, la notion de fabula établie dans la Généalogie n'est pas applicable au Décaméron.

I9. XIV, I6. Dans le chapitre 9 du livre XIV, pour illustrer l'utilité pratique des fabulae, Boccace soutient qu'elles ont déjà fait aimer l'étude et la connaissance à des esprits "qui étaient tombés dans le désœuvrement", et qu'elles ont comme réveillés : pour preuve, il raconte l'histoire du jeune Robert d'Anjou. Par ailleurs, il incite les calomniateurs, qui jalousent le succès des poètes, à se rendre eux-mêmes attrayants s'ils veulent être écoutés.

20. XIV, 7 : «[ce bouillonnement] oblige l'esprit à désirer écrire, il conçoit des inventions étrangères et inconnues, [...] il embellit la composition d'un tissu de mots et de pensées inhabituels [...].» (C’est nous qui soulignons.)

2I. "Intendiamo ora dimostrare che filoginia e misoginia, servizio alla corte d'amore e rifiuto della sua corte, non equivalgono a semplici cambiamenti limitati ad alcune idee e contenuti dell'opera del Boccaccio, ma presuppongono idee diverse della cultura, si collegano a fondamenti e prospettive che sono differenti nel loro complesso, e danno vita a due sistemi culturali distinti. Cambia il messaggio delle opere prodotte nelle due fasi della carriera intellettuale del Boccaccio, e con esso cambiano i presupposti ideologici e il pubblico al quale egli si rivolge; in una parola, cambiano le opere e l'intero sistema comunicativo in cui sono inserite e dal quale traggono significato." (F. Bruni, Boccaccio. L'invenzione della letteratura mezzana, Bologne, Il Mulino, I990, p. 35.) Bruni explique qu'il ne s'agit pas seulement de deux phases en succession dans la carrière de Boccace : en réalité, l'idée de la culture qui triomphera dans les œuvres de la maturité avait déjà intéressé le "premier Boccace» (ibid., p. 33-82). 
Bruni constate en effet que Boccace ne cite aucune de ses œuvres en langue vulgaire dans la Généalogie. Le topos de la modestie de l'auteur par rapport à ses propres œuvres n'explique pas pleinement ce silence, puisque Boccace cite en revanche son Buccolicum carmen - un texte qui relève du style humble ${ }^{22}$. Quant au choix de la langue vulgaire, bien que Boccace juge le latin plus noble que les langues romanes, il n'est pas à lui seul un motif convaincant, puisque l'auteur écrit que la connaissance totale du latin est nécessaire au poète, mais que «quelques-uns ont remarquablement écrit dans leur langue maternelle et rempli toutes les missions de la poésie» (XIV, 7). Il mentionne aussi plusieurs ouvrages en langue vernaculaire : au sujet de la Comédie, qu'il met au nombre des plus nobles ouvrages de poésie, et dont il cite même quelques vers traduits en latin, il précise qu' «en admirable artiste [Dante] [1'] a écrite en vers et en dialecte florentin" (XV, 6) ${ }^{23}$. Et lorsqu'il évoque Francesco da Barberino parmi ses sources modernes, Boccace émet un jugement très positif sur ses vers en langue vulgaire ${ }^{24}$.

Selon Francesco Bruni, le Décaméron appartient à une tradition narrative que Boccace a fondée à partir de sa lecture de la Vie nouvelle de Dante - celle précisément que ce critique a nommée letteratura mezzana. Cette tradition rassemble des ouvrages inspirés au poète par l'amour des femmes, qui en sont également les destinataires privilégiées; ces œuvres exaltent le sentiment amoureux et ne mesurent pas l'idéologie qu'elles véhiculent à l'aune des vérités les plus hautes. La seconde partie de la carrière intellectuelle de Boccace est dominée par une idée nouvelle de la culture : le champ de la littérature, s'ouvrant aux sujets les plus nobles, se fermerait aux intérêts qui avaient présidé à la rédaction des œuvres narratives de la jeunesse, en vertu d'une scission franche, caractéristique de la pensée du «second Boccace», entre le dévouement aux études et la dévotion à l'amour. L'inspiration ne devait plus venir que des Muses, qui appellent le poète vers les sujets les plus nobles, et les destinataires ne pouvaient plus avoir l'humilité des femmes. Le premier Boccace avait inauguré un registre médian de la littérature : sur une échelle de valeur à trois degrés, il plaçait ses propres œuvres narratives entre la littérature la plus noble, qui véhicule les vérités les plus précieuses, et les œuvres populaires, qui

22. F. Bruni, Boccaccio, op. cit., p. 5I.

23. Dans le livre XIV, lorsqu'il mentionne le poème de Dante, Boccace exprime l'idée qu'il s'agit d'une œuvre remarquable «si l'on accepte qu'il [l']ait rédigé[e], mais avec quel art! dans sa langue maternelle [...]" (XIV, 22).

24. XV, 6: «Bien qu'il ait connu le droit canon bien mieux que la poésie, il a tout de même fait paraître quelques livrets d'une poésie éclatante en dialecte vulgaire, qui attestent la noblesse de son esprit.» 
traitent de sujets plus humbles et sont composées sans savoir-faire. Selon Francesco Bruni, la typologie que Boccace établit dans le chapitre 9 du livre XIV de la Généalogie ne comporte véritablement que deux catégories : la littérature noble, qui traite dans un langage allégorique les sujets les plus élevés (philosophiques, théologiques), et une littérature dégradée, dans laquelle la lettre n'est le symbole d'aucune vérité plus élevée. Cette nouvelle classification est inapte à rendre compte des œuvres de jeunesse de Boccace, lequel éprouve un embarras certain lorsqu'il est confronté à sa première production narrative. Des œuvres telles que le Décaméron ne peuvent pas être incluses au sein de la littérature digne, car leurs contenus, qui ont la modestie des choses humaines, ne renvoient pas à des vérités plus hautes (on ne peut pas les lire comme des allégories : la lettre n'est pas le signifiant d'un signifié plus noble); nécessairement, écrit Bruni, ces œuvres se rapprochent dangereusement de l'autre pôle.

Notre lecture des derniers livres de la Généalogie nous incite à nuancer ce jugement. Il est certain que le projet du Décaméron tel qu'il est présenté dans le prologue et la conclusion de l'auteur est loin de coïncider parfaitement avec les aspirations de la Généalogie. La désignation des femmes comme destinataires privilégiées de l'ouvrage inscrit celui-ci dans le circuit culturel de la letteratura mezzana; pour ce qui est des contenus, ces deux passages insistent sur la centralité de la thématique amoureuse, presque comme si l'éventail des thèmes abordés dans les cent nouvelles se réduisait à ce seul sujet, assorti à l'esprit des destinataires - mais aussi de l'auteur. Cette prédominance supposée du thème pose deux problèmes : d'abord celui de la décence et de la moralité, ensuite celui de la sphère culturelle que les récits embrassent. L'auteur lui-même règle la question de la décence par l'affirmation du caractère naturel de la passion amoureuse (voir l'introduction de la quatrième journée) : puisque le désir fait partie du réel, la présence de ce sujet dans les récits est justifiée. Toutefois, on peut penser qu'aux yeux du Boccace de la Généalogie, l'auteur du Décaméron se montre parfois ou souvent, à l'instar d'Ovide, d'une complaisance suspecte à l'égard de la passion amoureuse et du désir charnel. D’une manière plus générale, il apparaît que dans le Décaméron, la position de l'auteur par rapport à ses nouvelles est ambiguë : toutes, sauf la nouvelle inachevée de l'introduction de la quatrième journée, sont mises à distance par la délégation de la narration aux "devisants»; et Boccace, dans la conclusion de l'auteur, souligne malicieusement l'ambiguïté de cette délégation. Nombreux sont les exemples de vice qui ne sont pas fustigés, à l'instar de l'histoire de Ciappelletto, et nombreuses sont les nouvelles suspectes d'être purement récréatives. 
La seconde question est plus vaste. Il est évident que, ne serait-ce que par la variété des thèmes abordés, le Décaméron dépasse en réalité le cadre de la letteratura mezzana. Mais on peut considérer que la désignation de l'amour comme thème principal du recueil revient à en faire le symbole de l'ensemble des sujets traités, qui relèvent tous de la sphère des choses humaines. Les nouvelles traitent des aspects variés de la vie terrestre dans son immanence. En cela, elles n'appellent pas une interprétation allégorique : leur lettre ne renvoie pas à un contenu qui la transcende et la placerait au second plan, comme dans l'allégorie traditionnelle ${ }^{25}$. Nous avons vu que, selon Francesco Bruni, les œuvres de jeunesse de Boccace, ne pouvant être lues comme des allégories in verbis, c'est-à-dire ne traitant pas per allegoriam de vérités plus hautes que celles désignées par la lettre, elles ne peuvent appartenir à aucune des trois premières catégories de fabula établies dans le chapitre 9 du livre XIV. Cet argument ne nous semble pas pertinent, dans la mesure où - limitant notre examen à la question du Décaméron - il nous semble que les nouvelles de Boccace, dans leur ensemble, relèvent de la troisième catégorie de récit : tout comme les fictions de Plaute et de Térence, elles ne recourent pas à l'allégorie, mais traitent du monde terrestre dans son immanence, en racontant des faits vraisemblables - elles ont une dimension cognitive, et donc une valeur poétique.

Par ailleurs, le prologue et la conclusion de l'auteur assignent aux nouvelles une fonction primaire de consolation (Prologue, 9-I5) - fonction également reconnue aux fabulae dans la Généalogie (XV, 9). La vertu consolatoire des récits découle du plaisir de la lecture et de l'utilité de celle-ci, car l'utilité (l'enseignement) est elle-même à l'origine du plaisir. Le caractère récréatif des nouvelles n'est pas disjoint de leur fonction cognitive, car leur lecture «[libère] l'esprit de sa souffrance immédiate, à travers une compréhension plus vaste de l'existence, que seule la poésie peut offrir ${ }^{26} »$. On n'est pas ici très éloigné des idées de la Généalogie, où le plaisir de la poésie découle de l'intelligence du sens plus que de la belle forme.

Toujours au sujet de la possible identification des nouvelles aux fabulae, une question de forme n'a pas été posée par Bruni. Au risque d'être taxé de trivialité, nous nous demandons si le fait que le Décaméron soit écrit en prose est susceptible de l'exclure du domaine poétique. Car si ces nouvelles

25. Francesco Tateo a fait remarquer à ce sujet que dans la Généalogie, Boccace n'emploie pas le terme allegoria, mais parle toujours d'integumentum et de velamen (F. Tateo, "Retorica» e "poetica", op. cit., p. II6-I60). 26. Ibid., p. I48. 
peuvent être assimilées, malgré l'altérité des modes narratifs (récit/drame), aux comédies latines, leur caractère d'œuvres en prose les en sépare - et les sépare de toutes les œuvres mentionnées à titre d'exemple (ou dont les auteurs sont nommés) dans les livres XIV et XV $\mathrm{XV}^{27}$. Peut-on estimer qu'en ne citant que des œuvres versifiées, Boccace ait simplement, incidemment, rendu compte de l'histoire littéraire (puisque dans la littérature latine, la fiction est généralement confiée au vers - ce qui reste vrai assez tard pour la littérature italienne, mais plus après Boccace)? Ce serait sans doute forcer un peu les choses.

On constate que dans l'ensemble, Boccace est moins disert que Pétrarque sur le vers comme caractéristique de la poésie. Une raison évidente de ce silence relatif est que les attaques des adversaires visent le contenu plus que la forme des poèmes : le propos est de montrer que les fabulae sont pleines de sens. Par ailleurs, les détracteurs de la poésie voient sa beauté formelle comme un danger (XIV, I6) : nul besoin donc d'exalter la séduction qu'exerce la musicalité du vers! Il reste qu’à deux reprises, Boccace mentionne la forme versifiée comme une qualité intrinsèque de la fabula. En effet, au chapitre I7 du livre XV, il affirme que, tandis que la philosophie s'écrit le plus souvent en prose, la poésie s'écrit en vers, car le poète est "extrêmement soucieux [...] de la forme rare et frappante de son texte». Comme on l'a vu, les enseignements de la poésie sont de l'ordre de la révélation : le vers, comme l'image nouvelle, contribue à renouveler le regard sur le réel. Les chapitres 7 et 8 du livre XIV sont consacrés à la naissance de la poésie, qui naît comme prière et dont le vers est un attribut primordial. Adressée au destinataire le plus digne, la prière doit s'éloigner par sa forme du langage quotidien : aussi la poésie naît-elle comme parole cadencée, détachée par ses qualités rythmiques de la trivialité des choses terrestres.

Toutefois, on se souviendra qu'au chapitre io du livre XIV, Boccace incluait aux nombre des fabulae signifiantes les histoires des vieilles femmes - d'humbles récits d'invention populaire, transmis par le canal de l'oralité et que l'on ne peut imaginer qu'en prose. Peut-être Boccace estime-t-il que les œuvres versifiées sont le fruit d'un savoir-faire plus raffiné que celles en prose, et qu'elles sont plus belles. Mais le fait est qu'il lui importe d'affirmer que l'invention poétique n'est pas la prérogative d'experts ni l'application de règles de composition préétablies, mais avant tout l'activité d'un esprit disposé à méditer sur son expérience du

27. Les auteurs cités sont Homère, Hésiode, Euripide, Solon pour la Grèce antique; Ennius, Plaute, Térence, Virgile, Horace, Juvénal, Perse, Stace, Claudien pour la Rome païenne; Juvencus, Prudence, Coelius Sédulius, Arator pour les latins chrétiens. 
monde - si culturellement modeste et si ignorant de l'art consommé des grands poètes soit-il ${ }^{28}$. Par ailleurs, on remarquera que la prose écrite se détache nécessairement du langage quotidien, et que la prose vernaculaire de Boccace n'est pas du tout dépourvue de rythme : l'usage du cursus notamment, en fait une expression cadencée.

Il apparaît que la réception du Décaméron par le second Boccace est complexe et ambiguë. La langue vulgaire, le choix du destinataire et le caractère prosastique des nouvelles le rangent probablement dans une catégorie modeste, sinon humble de poésie. Mais le projet cognitif d'embrasser le monde terrestre dans sa variété, dans une perspective "réaliste», reste recevable, et honorable, à l'aune de la Généalogie. Reste le problème de la «moralité» - un critère définitoire que Boccace soutient avec force dans sa défense de la poésie, mais au sujet duquel il ne semble pas être parvenu à une expression théorique parfaitement claire et univoque ${ }^{29}$.

\section{Décaméron, De insigni obedientia et fide uxoria (Pétrarque), Généalogie : modèles et préceptes pour la nouvelle}

Durant la première moitié du $\mathrm{Xv}^{\mathrm{e}}$ siècle, quelques grandes figures de l'humanisme ont cultivé le genre de la nouvelle. Tous n'ont pas consacré le même travail, le même temps, la même énergie, à l'écriture de nouvelles; tous ne l'ont pas abordée de la même manière. Certains, comme Leon Battista Alberti, ont écrit de nombreuses nouvelles regroupées en recueils. D'autres, tels que Leonardo Bruni, Bartolomeo Fazio ou Enea Silvio Piccolomini, sont les auteurs d'une nouvelle unique (ou de deux dans le cas de Bruni) : et ces textes représentent la seule incursion de ces auteurs dans le domaine de la fiction. Pour des lettrés tels que Leonardo Bruni ou Enea Silvio Piccolomini, qui travaillent à redécouvrir et se réapproprier la culture antique, s'essayer au genre de la nouvelle signifie se tourner vers la culture du Moyen Âge, qu'ils dénigrent par ailleurs et dont ils se sentent sortis $^{30}$, puisque la nouvelle est un genre roman, qui n'a pas à proprement parler de modèle antique, latin ou grec ${ }^{31}$. Cela signifie aussi intégrer, au

28. F. Tateo, "Retorica» e "poetica»..., op. cit., p. I23.

29. Voir note 17. Rappelons-nous par ailleurs qu’à la fin de sa vie, Boccace recopiait et retouchait son Décaméron dans le prestigieux codex Hamilton 90 de la bibliothèque de Berlin.

30. Les Dialogi de Bruni en sont un éloquent témoignage (L. Bruni, Dialogi ad Petrum Paulum Histrum, Florence, Olschki, 1994).

3I. Voir notamment P. Fedeli, "Modelli classici della novella italiana", dans La Novella italiana, Actes du Colloque de Caprarola (19-24 septembre 1988), Rome, Salerno, I989, p. 303-336. 
moins en partie, à leur canon littéraire presque exclusivement latin un modèle en langue vulgaire : le Décaméron. Or, la réception que l'élite humaniste du premier $\mathrm{Xv}^{\mathrm{e}}$ siècle fait du recueil est complexe et ambiguë, tout comme l'est sa perception de son auteur.

Dès avant sa mort, Boccace est largement considéré comme un père de la culture humaniste, à l'instar de Pétrarque, dont il a été l'ami, le correspondant et le collaborateur ${ }^{32}$. Les élèves de Boccace, puis les intellectuels issus du cercle de Salutati ont l'impression de vivre une époque nouvelle caractérisée par la renaissance de la culture. De ce renouveau, Boccace avait été un grand promoteur. Aussi ses biographes le célèbrent-ils comme le réintroducteur du grec à Florence et comme un maître et un guide pour les études antiques. Â sa mort, un an après celle de Pétrarque, Coluccio Salutati envoie une lettre à Francesco da Brossano dans laquelle il célèbre les deux hommes comme les deux luminaires qui ont éclairé la culture de leur époque ${ }^{33}$. Dans cette épître, Salutati fixe une liste canonique des œuvres de Boccace qui sera reprise par la majorité des biographes : il cite le Buccolicum carmen, le De montibus, le De casibus, le De mulieribus et la Genealogia deorum gentilium, c'est-à-dire uniquement la production latine. Cette liste réapparaît sous la plume de Franco Sacchetti, pourtant la voix de la culture vulgaire, puis, cinq lustres plus tard, dans la Vie de Manetti (I440) ${ }^{34}$. D’une manière générale, dans les écrits qui traitent de Boccace, soit les œuvres en langue vulgaire sont complètement passées sous silence, soit elles sont évoquées de façon générale, sans mention des titres. Dans sa "Notizia del Boccaccio ${ }^{35}$ ", Leonardo Bruni exprime l'idée - tout à fait opposée à l'opinion dominante - que Boccace se montre supérieur dans ses œuvres en langue vulgaire, notamment parce qu'il n'a jamais bien réussi à maîtriser le latin. C'est là un jugement très marginal. Comme on l'a vu, Salutati et Sacchetti ne mentionnent pas la production italienne de Boccace; Sacchetti leur préférait l'excellence des lettres latines, mais c'est surtout en vertu d'un moralisme affiché que la production vulgaire est dévalorisée : il reproche au jeune Boccace la lascivité de son inspiration. Manetti fait l'éloge des ouvrages en langue vulgaire, il en salue l'élégance, mais il disqualifie ces œuvres qui «ne sont au fond qu'une étape

32. Voir particulièrement G. Billanovich, Petrarca letterato: lo scrittoio del Petrarca, Rome, Edizioni di Storia e Letteratura, 1947, chap. 2 ("Il più grande discepolo»).

33. J. Bartuschat, Les "Vies" de Dante, Pétrarque et Boccace en Italie (XIVe-XVe siècles). Contribution à l'histoire du genre biographique, Ravenne, Longo, 2007, p. 193.

34. Ibid., p. 194-195.

35. Ce texte clôt les Vite di Dante e di Petrarca, que l'on peut lire dans L. Bruni, Opere letterarie e politiche, (P. Viti éd.), Turin, UTET, 1996. 
sur le chemin vers une culture accomplie, celle de l'humanisme latin ${ }^{36}{ }^{\prime}$. Les biographes de Boccace "estimaient visiblement que [sa] production vulgaire $[. .$.$] , en vers et en prose, ne pouvait rien apporter à la construc-$ tion de leur idéal culturel ", écrit Johannes Bartuschat ${ }^{37}$.

Plus tôt, Pétrarque avait évoqué sa fréquentation du Décaméron dans une lettre adressée à son auteur, et dont nous reparlerons. Pressé par des activités plus importantes, le "père de l'humanisme» n'a fait que parcourir cet ouvrage, entré en sa possession par il ne sait quel biais. Il salue l'évocation de la peste de 1348 et les passages dans lesquels Boccace défend ingénieusement ses nouvelles. Il écrit encore avoir été parfois offensé par certains récits trop lestes, mais cette liberté est due, selon lui, à la jeunesse de l'auteur et au circuit culturel auquel l'ouvrage appartient (le circuit de la littérature vulgaire, destinée à un public populaire, revendiquant un style humble et traitant de sujets légers). Dans l'ensemble, il juge le Décaméron comme une lecture plaisante, mais qui demeure en marge de la culture que les deux lettrés promeuvent à présent, ne serait-ce que par sa destination populaire et l'emploi de la langue vulgaire ${ }^{38}$.

Dans sa Vie de Dante, Bruni reproche à Boccace d'avoir abordé sa propre biographie du poète florentin dans la même disposition que lorsqu'il écrivait "ses dix journées d'amour» : à ses yeux aussi, le Décaméron pèche par légèreté.

Le culte de Boccace, on le voit, concerne l'auteur latin : pour diverses raisons, le Décaméron ne fait pas partie de ses titres de gloire. Malgré cela, c'est pourtant d'abord à travers ce recueil, modèle fondamental et ambigu, que la culture humaniste inclut le genre de la nouvelle à sa pratique littéraire, le texte fondateur étant la traduction de la nouvelle X, ro par Pétrarque, dont nous reparlerons.

À travers l'étude de quelques nouvelles ayant vu le jour dans les milieux humanistes du premier $\mathrm{Xv}^{\mathrm{e}}$ siècle, nous examinons maintenant comment la notion de fabula établie par Boccace d'une part, et d'autre part la réécriture de Décaméron X, Io par Pétrarque, ont conditionné la réception du modèle décaméronien et, plus largement, la pratique de la nouvelle chez les humanistes.

D’une façon générale, on constate que les caractéristiques formelles du récit bref en prose sont de nature à favoriser la réalisation d'un projet d'écriture justifié comme dans la Généalogie par la fonction didactique du

36. Ibid., p. 205

37. J. Bartuschat, Les "Vies", op. cit., p. I95.

38. Pétrarque, Lettres de la vieillesse, XVII, 3. 
récit. La brièveté de la forme «nouvelle» a pour effet principal d'inciter à la relecture, en renforçant la faculté qu'a le récit de se placer en continuité avec les expériences vécues du lecteur, et, le cas échéant, en assurant la longévité d'un message. Par sa condensation, la nouvelle offre au lecteur la possibilité de tenir à l'esprit la plupart des éléments du récit, dont ressortent d'éloquents effets de structure : la condensation favorise l'intelligence du sens du récit et la méditation sur ce sens. Une autre caractéristique de la forme «nouvelle» est de ne pas instaurer l'illusion d'une totalité : les personnages peu nombreux, la chronologie souvent réduite, la caractérisation élémentaire des espaces sont autant d' "éléments épars [qui émergent] sur fond de néant ${ }^{39}$ ". "Nous entraînant dans le mouvement d'une création démiurgique, le roman nous fait oublier la réalité autour de nous, quand la nouvelle nous y renvoie [...]", écrit Jean-Pierre Aubrit ${ }^{40}$; peut-être plus que tout autre genre, donc, la nouvelle "provoque une résonance féconde dans l'esprit du lecteur ${ }^{41}$ ». Peut-être peut-on considérer aussi que l'usage de la prose, plus proche que le vers du langage quotidien, favorise la capacité de la fiction à s’insérer dans la continuité des activités du negotium.

Comme nous y avons fait allusion, la traduction de Décaméron X, Io - la nouvelle de Griselda - par Pétrarque (1373-I374) constitue un précédent à l'écriture de la nouvelle en latin, refondant ce genre narratif. Gabriella Albanese a montré comment cette traduction-adaptation autorise l'inclusion partielle du modèle décaméronien au corpus littéraire des humanistes, et ouvre la voie à leur pratique du genre de la nouvelle ${ }^{42}$. Non seulement cette version latine constitue un exemple pratique, mais Pétrarque trace aussi une ligne de démarcation entre les nombreuses iocosa et laevia et les quelques pia et gravia, plus honorables que les premières (Lettres de la vieillesse, XVII, 3); et cette distinction aura une importance considérable pour les traductions latines postérieures du Décaméron.

Du recueil de Boccace, Pétrarque extrait donc une nouvelle qu'il juge différente de toutes les autres (Lettres de la vieillesse, XVII, 3) : c'est la dernière de la dernière journée - journée consacrée aux exemples de vertu. Il abolit du même coup le récit-cadre, la médiation des "devisants» et la mise à distance du récit par son auteur, caractéristique du Décaméron. Pétrarque envoie en effet sa Griselda latine (De insigni obedientia et fide uxoria) à Boccace dans une série de lettres (Lettres de la vieillesse, XVII, 3

39. J.-P. Aubrit, Le Conte et la nouvelle, Paris, Armand Colin, 2006, p. I5I.

40. Ibidem.

4I. Ibid., p. I50.

42. G. Albanese, «Da Petrarca a Piccolomini. La codificazione della novella umanistica», dans Favole Parabole Istorie, Actes du Colloque de Pise (26-28 octobre 1998), Rome, Salerno, 2000. 
et 4). Le Décaméron ménageait une place à l'interprétation des récits : les marges des nouvelles étaient le lieu d'un commentaire, d'un dévoilement partiel du sens (ou, plus souvent, des sens) par un ou plusieurs devisants. Le modèle «lancé» par Pétrarque en fait au contraire le lieu d'expression d'un exégète dont le commentaire fait autorité : l'auteur, dont l'interprétation oriente la lecture, tendant à prescrire un protocole de lecture per allegoriam.

Dans le Décaméron, c'est le personnage de Dioneo qui raconte l'histoire de Griselda : les derniers mots du devisant subvertissent la dimension exemplaire du récit ${ }^{43}$, brouillant les pistes de l'interprétation de cette nouvelle qui demeure profondément ambiguë. Pétrarque expurge le récit de ce passage (l'interprétation politique vient d'ailleurs à disparaitre), mais joint en revanche un commentaire personnel, dont l'écriture reflète le caractère familier de la correspondance amicale (Lettres de la vieillesse, XVII, 4).

Dans ce texte, le traducteur justifie son entreprise par la dimension parénétique de la nouvelle, qui agit à deux niveaux. Pétrarque nie que l'attitude de Griselda puisse servir de modèle aux épouses contemporaines, qui n'ont pas la vertu de la femme de Gualtieri : malgré l'emploi de ce topos misogyne de la littérature édifiante, l'exégète indique bien un premier niveau de lecture. Mais la patience du personnage est surtout un exemple adressé aux chrétiens; en vertu de l'isomorphie de deux relations et de deux situations (et peut-être aussi en vertu du développement et de l'extension poétique de la notion de mariage mystique), Pétrarque rapproche les épreuves que le marquis inflige à son humble épouse de la mise à l'épreuve des âmes par Dieu ${ }^{44}$. Son commentaire révèle le sens moral du De obedientia, dont il fait un récit «doublement» exemplaire. La seconde interprétation sous-entend une lecture per allegoriam, dans laquelle le sens littéral est le signifiant d'un signifié relevant du domaine spirituel, plus noble, de la vie de l'âme chrétienne, de la Foi : l'allégorie fait du De obedientia une sorte de "Griselda moralisée», version latine

43. Déc., X, Iо, 68-69: "Que dira-t-on de cela, sinon que même dans les pauvres chaumières descendent parfois du ciel des âmes divines, tout comme il y a, dans les demeures royales, des gens qui seraient plus dignes de garder les cochons que de gouverner les hommes? Qui d'autre que Griselda aurait pu, non seulement sans verser de larmes, mais d'un air joyeux, endurer les épreuves cruelles et inouïes que lui imposait Gualtieri? Peut-être celui-ci aurait-il mérité de tomber sur une femme qui, étant chassée en chemise, se serait fait secouer le poil par un autre, et cela aurait été une bonne chose. " (Les citations en français du Décaméron sont tirées de Boccace, Décaméron, traduction, introduction et notes sous la direction de C. Bec, Paris, Librairie Générale Française, 1994.)

44. Cette interprétation repose notamment sur l'association de la noblesse sociale du mari et de la noblesse absolue de Dieu d'une part, et d'autre part de l'humble extraction sociale de l'épouse et de l'humilité absolue de l'âme humaine. À la lumière de cette lecture, on comprend que l'interprétation politique que faisait Dioneo de la nouvelle $\mathrm{X}$, Io devait disparaître de la version latine. 
apte à figurer dans les recueils d'exempla des prédicateurs, à l'instar des Métamorphoses ovidiennes, que l'exégèse ecclésiastique avait conciliées, au prix d'audacieuses transpositions, avec la théologie chrétienne.

La lecture de Pétrarque tend à ramener la nouvelle dans le giron dont le premier Boccace l'avait sortie : le De obedientia et fide uxoria signe un retour à la logique édifiante de l'exemplum chrétien. Certaines intercenales du jeune Leon Battista Alberti ne s'éloignent pas tellement de cette conception de la fonction exemplaire du récit bref. C'est le cas par exemple de la nouvelle Amores, qui date probablement de la fin des années vingt ou du début des années trente du $\mathrm{Xv}^{\mathrm{e}}$ siècle.

Un prologue métadiégétique précède le récit. L'auteur y annonce le sujet : les amours d'un jeune homme prometteur dans le domaine des études nommé Friginnius. Il justifie le choix de ce sujet par l'utilité qu'en tireront les lecteurs. Alberti souligne la dimension pédagogique de la nouvelle en mobilisant largement le champ sémantique de l'étude et de l'enseignement. En énonçant la leçon de vie que le lecteur devra tirer du récit, il lie à la connaissance du cas raconté une norme de comportement à tenir. Il ne fait pas de doute à ses yeux que l'histoire de Friginnius suscitera de la pitié, et la crainte et le dégoût de la passion amoureuse, tout en dévoilant les noirceurs de l'âme féminine : de la fréquentation de ces êtres pervers il conviendra que les sages se gardent, ayant bien compris que l'amour conduit à la ruine les esprits les plus virils et les plus vertueux. Cette intercenalis se présente donc comme l'illustration d'une thèse. Le récit lui-même a un caractère démonstratif prononcé. La posture moraliste et pédagogique que le narrateur adopte en introduction se vérifie tout au long du récit, qui raconte comment un jeune homme prometteur participe impuissant à la ruine de son existence à cause de l'amour. La diégèse est entièrement soumise à la thèse qu'Alberti entend illustrer. L'expérience amoureuse est présentée comme totalement négative, comme une déchéance physique et morale, et comme une suite de souffrances infligées à l'amant par sa maîtresse. Le récit de ces malheurs est rythmé par des commentaires du narrateur, qui s'inscrivent dans le prolongement idéologique de l'introduction puisqu'ils soulignent la déchéance du personnage principal.

Cette intercenalis se présente donc comme une nouvelle argumentative : le récit censé relater des faits avérés (c'est un lieu commun de la tradition de la nouvelle en langue vulgaire) est appelé dès sa préface à démontrer la validité d'une thèse. Elle s'éloigne en cela du modèle de la nouvelle décaméronienne, que les "devisants» justifiaient invariablement 
par son caractère didactique et argumentatif, mais qui n'était jamais subordonnée à cette dimension.

La dimension argumentative d'Amores est évidemment fonction de sa vocation édifiante. Sous la plume d'Alberti, la nécessaire justification didactique de la fabula prend la forme d'une sorte d'exemplum laïque, adressé par un jeune humaniste à ses semblables afin de les mettre en garde contre les dangers de la passion ${ }^{45}$, et dont l'exégèse précède le récit et lui est mêlé. Le récit est justifié par son sens moral, mais l'auteur ne prévoit pas qu'il soit soumis à une lecture allégorique; la portée édifiante ne se joue pas sur le plan des choses divines, mais sur le plan des humana: les lecteurs sont exhortés à une vertu terrestre - la dévotion profane aux études.

Dans les années trente $\mathrm{du} \mathrm{Xv}^{\mathrm{e}}$ siècle, le diptyque de nouvelles dont Leonardo Bruni est l'auteur met également au premier plan la dimension exemplaire des récits brefs, mais d'une manière très différente d'Alberti ${ }^{46}$. Si Bruni adopte la typologie épistolaire d'ascendance pétrarquienne, l'exégèse d'auteur est absente du billet introductif : l'auteur s'y limite à souligner le fait que les deux fabulae se répondent par opposition (per opposita). Une partie de sa missive ne nous étant pas parvenue, on ignore si elle présentait comme son modèle pétrarquien une postface tenant lieu de commentaire. En revanche, il apparaît clairement que Bruni a exploité les ressorts formels propres à la nouvelle aux fins de réaliser une ouvre didactique. Sous sa plume, les effets de structure que favorise la condensation de la forme brève sont fonctionnels à l'intention didactique.

45. De la thèse que le récit véhicule, à savoir l'incompatibilité des études humanistes et de la passion amoureuse, Alberti se fait le chantre dans un écrit théorique sur la mission et le statut de l'intellectuel - le Des avantages et des inconvénients des lettres (I432) -, où le refus de l'amour profane était un trait définitoire de l'homme de lettres. Sur cette voie, Alberti recueille un certain héritage de Pétrarque et de Boccace, qui avaient soutenu la même position. Si le jeune Alberti ne conçoit pas la figure de l'intellectuel de la même manière que Pétrarque ou Boccace au siècle précédent (ses domaines d'étude, notamment, diffèrent), il emprunte tout de même des traits fondamentaux au portrait du poète que Boccace trace dans la Généalogie des dieux païens (nous pensons notamment à sa pauvreté et à sa solitude, rendues nécessaires par la dimension spirituelle de son travail et ses qualités morales). Par ailleurs, un autre texte a pu constituer une source d'inspiration privilégiée : le Corbaccio, récit de fiction que son auteur présentait déjà comme un "humble traité».

46. Il existe deux éditions récentes de l'épître à Bindaccio de' Ricasoli : l'une a été établie d'après l'incunable XV. IV. II9 de la Bibliothèque nationale de Turin, daté de I472 environ (M. L. Doglio, L'exemplum nella novella latina del Quattrocento, Turin, Giappichelli, 1975, p. I50; traduction italienne en regard); l'autre est tirée du manuscrit Magliabechiano IX 2 de la Bibliothèque centrale de Florence (M. Martelli, "Considerazioni sulla novella spicciolata", dans La novella italiana, op. cit., p. 215-243, p. 216). Nicoletta Marcelli a édité la nouvelle de Séleucos dans "La novella di Seleuco e Antioco. Introduzione, testo e commento", Interpres, XXII, 2003, p. 7-I83, où elle cite intégralement la lettre introductive à Ricasoli, en suivant le texte du Magliabechiano. On peut lire la réécriture de Déc., IV, i dans M. L. Doglio, L'exemplum..., op. cit., p. I52-I60. 
La lettre latine que Bruni adresse à son ami Bindaccio Ricasoli en I437 (ou I438, ou I43947) associe deux nouvelles, que l'auteur qualifie de fabulae. La première est une version latine de la nouvelle IV, i du Décaméron (la nouvelle de Tancrède, une nouvelle que sa teneur place au nombre des pia et gravia); le second panneau du diptyque consiste en une nouvelle "originale» en langue vulgaire - nouvelle que la critique nomme "de Séleucos». Le diptyque tel que nous le connaissons est le fruit d'un travail éditorial : la traduction de la nouvelle de Tancrède est celle de Bruni, tandis que la nouvelle en langue vulgaire est généralement attribuée à un auteur anonyme. Mais le projet d'accoler ces deux récits en un édifice narratif bilingue est bien de Leonardo Bruni, comme en témoigne la lettre introductive, dont l'attribution n'est pas mise en doute par la critique ${ }^{48}$.

La configuration en diptyque implique un rapprochement thématique et structural des deux nouvelles, mettant en lumière la primauté de l'intention didactique. En effet, le diptyque met en scène deux figures paternelles, Tancrède et Séleucos, confrontées à des situations en partie similaires : leur enfant brûle d'un amour interdit, mais que les pères ont la possibilité de satisfaire. La nouvelle de Tancrède décrit les conséquences funestes de la brutalité du prince, tandis que celle de Séleucos montre la générosité dont le roi de Syrie fit preuve envers son fils, et ses effets positifs. La nouvelle de Tancrède révèle la force destructrice de l'amour insatisfait, suivant un schéma récurrent dans la quatrième journée du Décaméron - dont chacune des nouvelles à l'issue funeste, plusieurs fois sanglante, forme une séquence avec la nouvelle inachevée de l'introduction. Sachant pour sa part que l'amour de son fils pour Stratonice s'avérera fatal, Séleucos donne à la passion d'Antiochos une forme nouvelle - celle de l'hymen - au prix d'un sacrifice qui se révèle finalement léger. Le désir potentiellement destructeur s'inscrit alors dans la logique communautaire de la construction de la cité terrestre. Il fructifie dans ce cadre du mariage, dans lequel l'amour acquiert une utilité et une valeur sur les plans économique et politique. L'histoire de Séleucos et de son fils

47. Les manuscrits ne concordent pas sur l'année de la rédaction. Une brève synthèse existe sur la question : V. Branca, «Un 'lusus' del Bruni cancelliere: il rifacimento di una novella del Decameron (IV, I) e la sua irradiazione europea", dans Leonardo Bruni cancelliere della Repubblica Fiorentina, Florence, Olschki, I990, p. $207-226$, n. 2.

48. Sur la question de l'attribution (ou non) de la nouvelle de Séleucos à Leonardo Bruni, voir M. Martelli, "Considerazioni sulla novella spicciolata», art. cité; Idem, «Il Seleuco, attribuito a Leonardo Bruni, fra storia e elegia", dans Favole, Parabole, Istorie, le forme della scrittura novellistica dal Medioevo al Rinascimento, actes du colloque de Pise (26-28 octobre 1998), Rome, Salerno, 2000, p. 23I-255; L. Bartoli, "Note filologiche sulle novelle spicciolate del Quattrocento", Filologia e critica, XX, 1995, p. 3-43; N. Marcelli, "La novella di Seleuco e Antioco. Introduzione, testo e commento", art. cité. 
Anthiocos confirment la thèse de la quatrième journée du Décaméron et la dépassent dans une perspective exemplaire.

Par ailleurs, la nouvelle de l'Anonyme (celle de Séleucos) a une structure circulaire très marquée. L'histoire débute par le mariage du roi et se clôt avec celui de son successeur. La circularité du récit confirme la thèse selon laquelle l'attitude vertueuse des personnages rend possible la perpétuation d'un monde stable à travers l'institution du mariage. La célébration de cette institution, l'exaltation de la famille, sont caractéristiques de la culture florentine de cette époque ${ }^{49}$. La nouvelle de l'Anonyme est le véhicule d'une idéologie que Leonardo Bruni avait déjà exprimée dans sa biographie de Dante. La structure de ce récit vient donc appuyer la thèse du premier concepteur du diptyque.

Un peu plus tardive, la longue Historia de duobus amantibus d'Enea Silvio Piccolomini est insérée dans une lettre : selon le modèle pétrarquien, elle est donc inscrite dans un dispositif liminaire destiné à en orienter la lecture. Dans les passages introductif et conclusif de l'épître que Piccolomini adresse à son ami Mariano Sozzini en I444, l'auteur assène une «morale» univoque de son récit : cette histoire d'amour tragique, censée dévoiler les dangers de la passion, est présentée par son auteur comme une mise en garde. En réalité, cette nouvelle (c’est-à-dire le récit et son paratexte) revient à une duplicité du discours caractéristique du modèle décaméronien. Comme nous l'avons déjà indiqué, les devisants de Boccace présentent invariablement leurs nouvelles comme l'illustration d'une loi (morale, psychologique, de comportement), mais le rapport entre la thèse énoncée et la narration s'avère toujours ambigu, le récit n'étant en fait pas subordonné à sa fonction argumentative. De même, il y a toujours un décalage entre le récit et les commentaires volontiers moralisants des «devisants». De manière semblable, la leçon assez plate et conventionnelle sur laquelle Piccolimini clôt sa nouvelle n'épuise nullement le sens du récit, et ne semble rendre compte de la narration que de façon très partielle. Il pourrait alors s'agir d'une "fausse leçon", appelée en réalité à renvoyer le lecteur au récit lui-même, d'une invitation à s'approprier le texte, à en sonder les sens multiples, ou les facettes contradictoires du sens le plus plein. Au moment où il semble imposer une interprétation unique et univoque, Piccolomini renvoie en réalité à la polysémie du récit : la

49. De nombreux ouvrages témoignent de la reconnaissance de l'utilité et de la dignité de la famille sur les plans privé et public, c'est-à-dire éthique, économique et politique, au cours du premier $\mathrm{Xv}^{\mathrm{e}}$ siècle : les livres De Familia d'Alberti, ou le Dialogue consolatoire de Manetti en sont deux exemples connus. 
subversion du modèle "édifiant» se réalise à travers la récupération de la duplicité du discours caractéristique du Décaméron.

Cette conception de la fiction échappant à l'interprétation totalisante, de la fiction qu'en tout cas on ne peut pas ramener à une morale lapidaire, n'est en fait pas opposée à la poétique (ou devrait-on dire aux poétiques) que Boccace développe dans la Généalogie. Cette idée est thématisée dans le chapitre qui traite de l'obscurité de certaines fictions (XIV, I2). Alors qu'en plusieurs lieux, Boccace déclare que l'intention de l'auteur ( intentio») est décisive pour le sens profond du récit ${ }^{50}$, il nuance ici son propos sur l'origine de la signification profonde des fabulae. Il convoque notamment l'autorité d'Augustin en citant un passage de son commentaire du psaume CXXVI (vers II) :

[Ce passage] est rédigé de façon assez obscure peut-être pour produire plusieurs interprétations, et pour que les hommes qui l'ont trouvé fermé, après l'avoir ouvert de plusieurs façons, repartent plus riches que s'ils l'avaient trouvé déjà ouvert.

Lorsqu'il souligne la polysémie des psaumes, Augustin ne précise pas de quelle latitude jouit le lecteur, dans quelle mesure sa lecture élabore des sens nouveaux et dans quelle mesure elle recueille, ou actualise, des sens préexistants. Boccace, qui applique à la poésie profane ce qu'Augustin dit de la poésie de la Bible, ne fait pas coïncider le sens avec l'intention de l'auteur : il le situe entre l'intention auctoriale et l'interprétation du lecteur. Aussi est-il naturel, par exemple, que le sage et l'ignorant ne tirent pas le même fruit de la lecture d'un même texte :

Les fables sont d'une telle importance que dès la première ligne les ignorants y prennent du plaisir et que l'esprit des savants s'y prépare aux choses cachées [...]. (XIV, 9)

La même idée apparaissait dans le passage où Boccace traite des récits faits aux veillées : le conteur poursuit des buts variés, ne cherchant pas à produire le même effet sur des auditeurs de sexe, d'âge et de condition variées (XIV, Io). Dans le chapitre 9 du livre XIV, pour convaincre de l'utilité des fabulae, Boccace souligne aussi le caractère déterminant que revêtent les circonstances de la lecture pour la prédominance de telle ou telle fonction du récit : pour l'homme d'État, la fiction peut être essentiellement

50. Voir par exemple le chapitre 9 du livre XIV : «Ils pourraient nous objecter que ce don doit servir à des desseins utiles et non futiles, or les fables sont futiles. Ce que l'on ne pourrait nier, si le poète projetait de composer de simples fables. Mais nous avons montré depuis longtemps que le manteau des fables signifie autre chose que < ce que dit > son écorce. C'est pourquoi certains ont l'habitude de définir ainsi la fable : «La fable est un discours à valeur exemplaire ou démonstrative dans une fiction, où, l'écorce une fois enlevée, apparaît l'intention du fabuliste." (C'est nous qui soulignons.) 
une distraction, l'occasion d'une véritable récréation des forces de l'esprit; pour le prisonnier innocent, l'histoire de Psyché - personnage victime de la jalousie des dieux, mais dont les aventures ont une issue heureuse - a une vertu consolatoire ${ }^{5 \mathrm{I}}$.

Dans le Décaméron, le récit de fiction n'avait pas besoin d'être justifié par son utilité pratique; dans la Généalogie, sa fonction didactique est officiellement primordiale. Mais plusieurs lustres après la rédaction du Décaméron, il apparait que pour Boccace, la fiction demeure ouverte à une pluralité d'interprétations, qui dépendent du texte lui-même, du contexte de sa réception ainsi que de la forma mentis de chaque lecteur - idée que Piccolomini (lecteur admiratif du Décaméron, comme en témoigne sa correspondance) partage, et dont il trouve peut-être l'expression théorique dans la Généalogie.

Par ailleurs, Piccolomini traite indirectement de la question théorique de la dimension cognitive de la fiction, qu'il met en regard de celle du récit de faits avérés. Dans l'introduction de la lettre à Sozzini, Piccolomini affirme qu'il s'apprête à mettre en forme une matière qui relève de faits qui se sont réellement produits, et non pas à raconter ce qui pourrait se passer en vertu de quelque probabilité ou de quelque nécessité, ce qui est l'œuvre du poète. Or, l'histoire des amours d'Eurialus et Lucretia est une réécriture - une proximisation, selon la terminologie de Gérard Genette ${ }^{52}-\mathrm{du}$ chant IV de l'Enéide, c'est-à-dire d'un récit dont le caractère fictionnel avait été démontré par Boccace. Pourtant l'auteur présente son œuvre comme tout ce que le chant IV n'est pas : le récit de faits récents et s'étant déroulés dans la ville même du destinataire, Sienne. La capacité de l'Historia (titre éloquent!) de toucher ses lecteurs et d'être entendue comme contenant un enseignement repose là-dessus. Car qui croirait qu'un récit vieux de mille cinq cent ans puisse parler avec pertinence du présent?

Mais la relation que le récit entretient avec l'Énéide ne pouvait échapper aux latinistes lecteurs de l'Historia, et on ne peut imaginer que Piccolomini ait voulu ou cru la leur dissimuler : à la lecture de ce récit qu'on leur présente comme historique, les lecteurs reconnaîtraient sous son travestisse-

5I. "Quand les princes sont pris par d'importantes affaires et qu'ils ont décidé de grandes mesures pour améliorer leur royaume, voulant redonner du nerf à leurs forces épuisées, à croire que la nature le leur enseigne, ils font venir des gens qui par leurs joyeuses fabulations refont leur âme épuisée. À ceux qui peinent sous le poids de l'adversité, les fables apportent parfois de la consolation, comme on le voit chez Apulée : on y lit que Carithée, une vierge généreuse, qui avait été capturée pour son malheur par des brigands et qui pleurait sa captivité, fut quelque peu réconfortée par la fable de Psyché qu'une vieille femme lui raconta plaisamment. " Notons au passage que c'est en vertu du même processus d'identification que les nouvelles d'amour du Décaméron sont susceptibles d'avoir une vertu consolatoire sur les lectrices (Prologue, 7).

52. G. Genette, Palimpsestes, la littérature au second degré, Paris, Seuil, 1982. 
ment l'archétype de l'amour tragique - le récit virgilien du peregrinus amor. L'auteur, en feignant de raconter des faits récents, renouvelle un topos du genre de la nouvelle et feint que des amours réellement advenues coïncident avec le récit de Virgile et témoignent de la véracité du mythe. Cette feinte n'échappe pas à ses lecteurs, qui sont en réalité conviés à constater que, si l'histoire d'Eurialus et Lucretia est vraisemblable, c'est bien qu'est demeurée intacte la valeur cognitive de la fabula virgilienne - c'est-à-dire d'une œuvre d'imagination que, précisément, Boccace avait prise en exemple dans le livre XIV de la Généalogie, pour justifier par un commentaire la légitimité de la liberté d'invention du poète, et, du même coup, illustrer la dimension cognitive de la fiction littéraire ${ }^{53}$.

Forts du modèle pétrarquien, Leonardo Bruni et Leon Battista Alberti ont donné au récit bref la mission didactique et édifiante que Boccace exaltait au sujet des fictions en vers. L'utilité pratique de leurs nouvelles, toutefois, ne se joue pas comme chez Pétrarque sur le plan des divina, mais sur celui des réalités terrestres, l'allégorie laissant la place au simple integumentum. Des récits exemplaires donc, mais qui s'en tiennent, comme les nouvelles du Décaméron, aux humana - au domaine de la "théologie naturelle» que la Généalogie indiquait comme le domaine de prédilection du poète, et plus particulièrement à l'existence humaine. Les nouvelles de Bruni et l'intercenalis Amores s'éloignent du modèle décaméronien dans la mesure où leur écriture est manifestement conditionnée par la volonté de délivrer un enseignement assez explicite. Sous leur plume, le récit-cadre et la médiation des «devisants» disparaissent, abolissant la mise à distance des récits par leur auteur. Par ce biais, ces auteurs manifestent et revendiquent l'adéquation du récit bref aux exigences didactiques et morales de la fabula telle que l'humaniste Boccace la défend. Subvertissant subtilement le modèle du De obedentia, Enea Piccolomini distingue l'une de l'autre les dimensions cognitive et parénétique de la fiction : il exalte la première par le jeu du travestissement de la fable et ravale la seconde au rang de cliché un peu creux, restituant au genre du récit bref son ouverture à des interprétations multiples.

53. XIV, I3. Des calomniateurs, pour prouver que les poètes étaient menteurs, alléguaient que Virgile avait falsifié l'histoire de Didon : "Ce qu'ils reprochent à Virgile est faux. Ce sage n'a pas voulu raconter l'histoire de Didon. En effet, il était parfaitement informé des faits; il savait que Didon, femme d'une honnêteté remarquable, avait mieux aimé mourir de sa propre main que de souiller dans de secondes noces la volonté de chasteté qu'elle logeait en son cœur. Mais voulant que l'artifice d'une voile poétique servît le propos de son œuvre, il composa une fable en de nombreux points semblable à l'histoire de Didon : c'est un droit, je l'ai dit un peu plus haut, qu'une vieille coutume accordait aux poètes.» (C'est nous qui soulignons.) 
Du De obedientia et fide uxoria de Pétrarque au De duobus amantibus de Piccolomini, l'évolution de la nouvelle humaniste semble bien emprunter un parcours en partie semblable à celui de la nouvelle en langue vulgaire : du récit bref où la fonction didactique justifie la fiction, comme dans l'exemplum et le conte, à la "nouvelle» à proprement parler - la nouvelle stricto sensu - qui demeure ouverte à de multiples interprétations, caractérisée qu'elle est par la mise à distance du récit. Et il semble bien que cette mise à distance, qui caractérise en premier lieu le Décaméron, n’ait jamais été pleinement "parjurée" par l'auteur de la Généalogie - ce qui constitue un nouvel élément de compréhension de la première réception du recueil par un humaniste, à savoir, par son propre auteur, Boccace, à quelques lustres de la rédaction du Décaméron, alors qu'il recopiait ce texte, en introduisant de nouvelles variantes, dans le manuscrit Hamilton 90. 